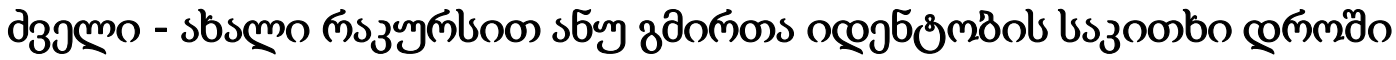

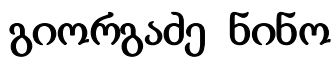

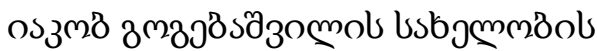

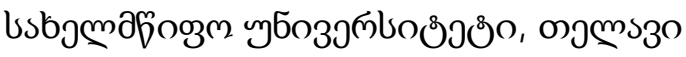 \\ https://doi.org/10.52340/idw.2021.518
}

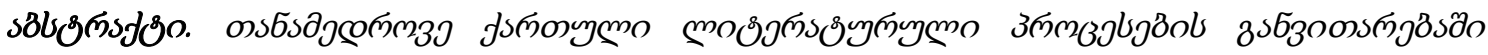

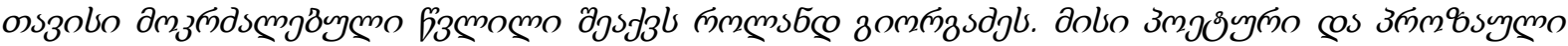

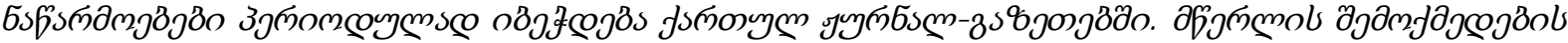

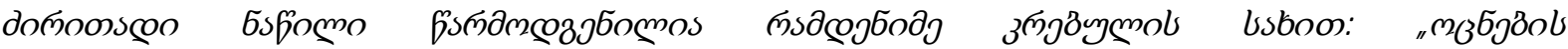

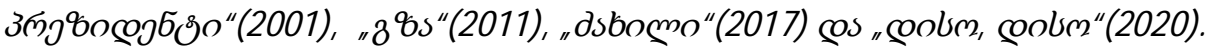

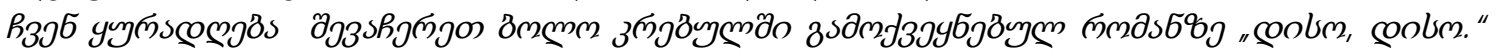

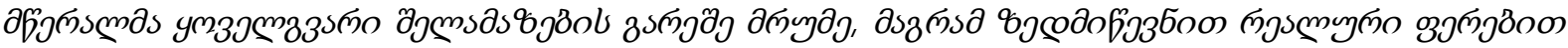

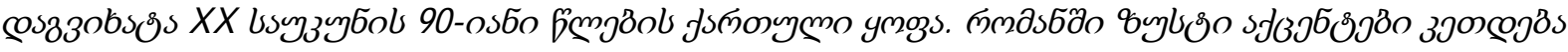

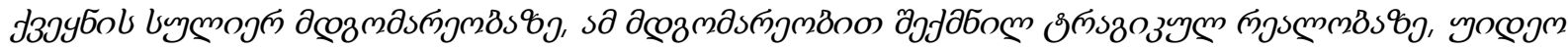

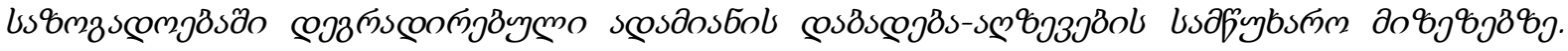

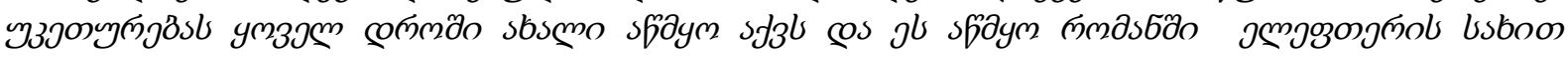
земобеj8s.

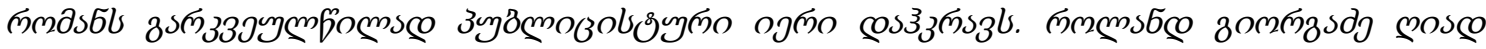

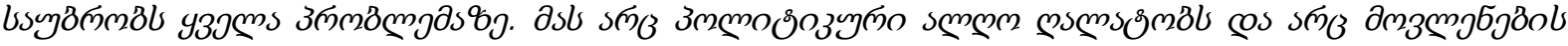

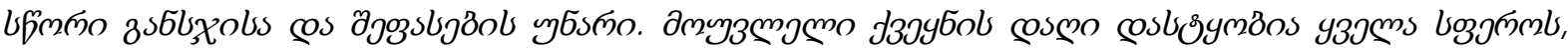

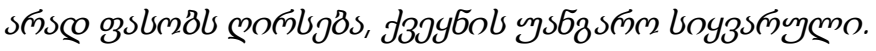

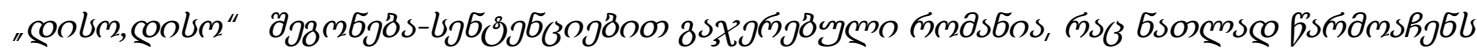

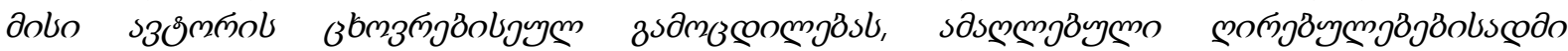

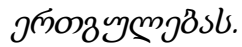

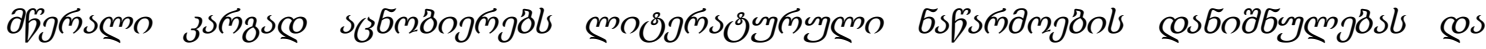

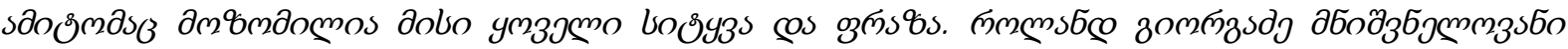

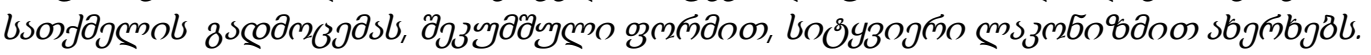

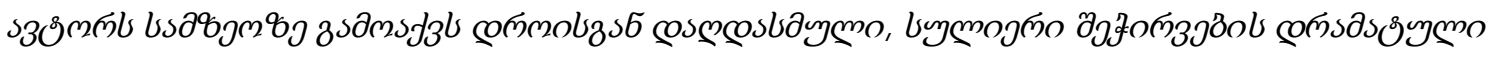

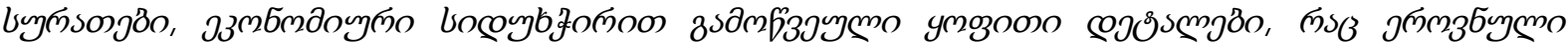

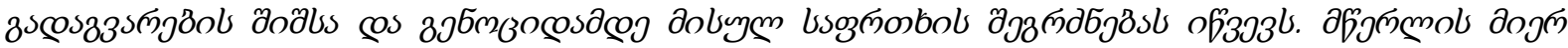

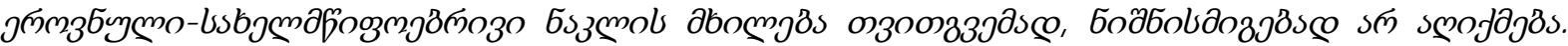

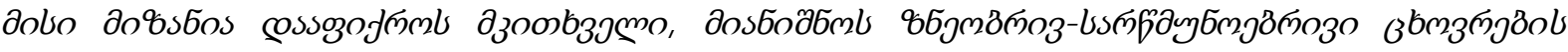

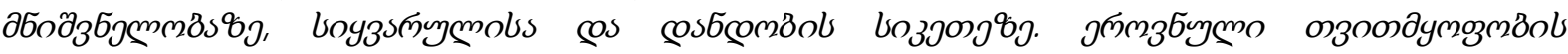

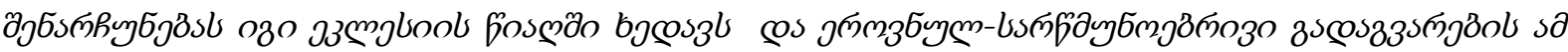

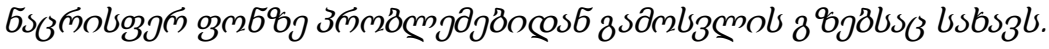

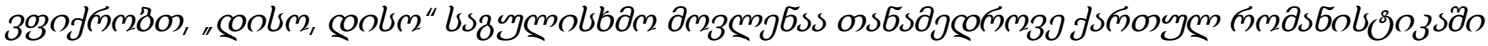

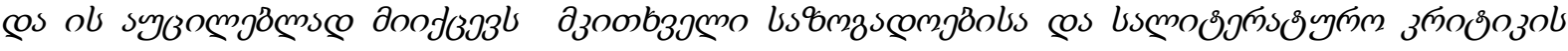

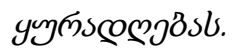

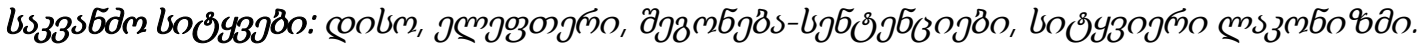

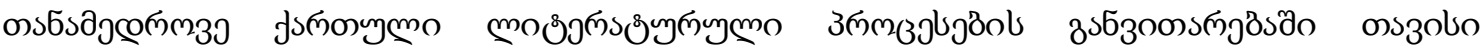

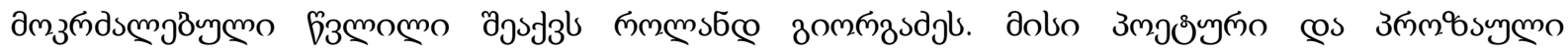

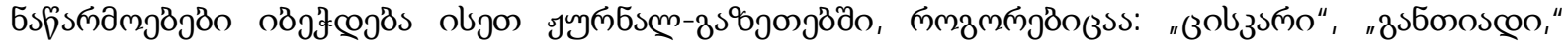




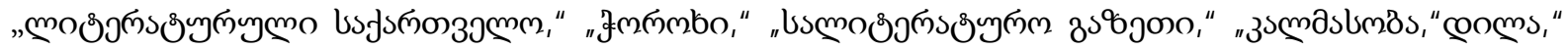

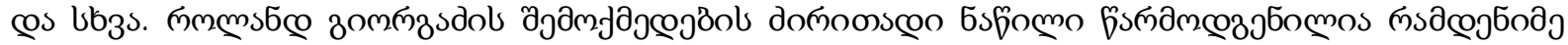

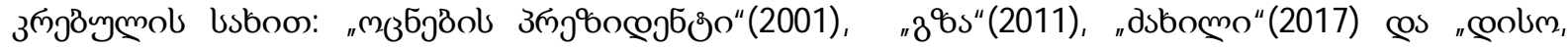
colvm"(2020).

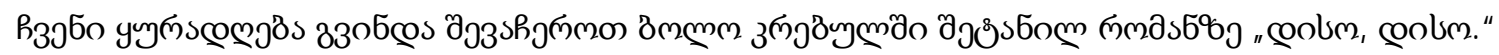

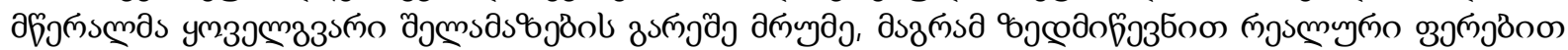

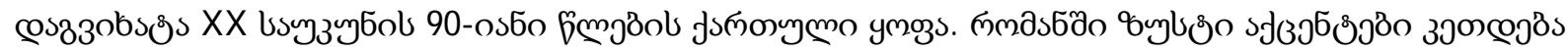

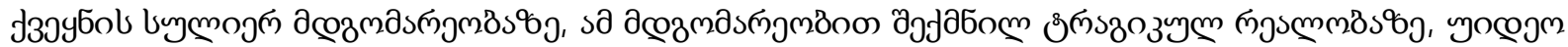

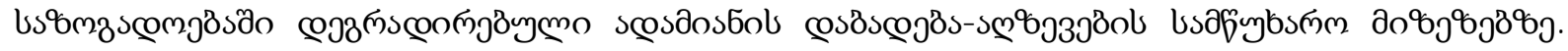

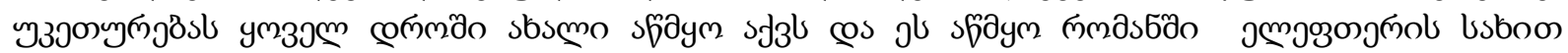
змобеgठs.

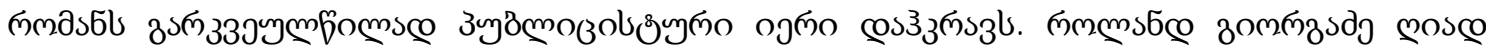

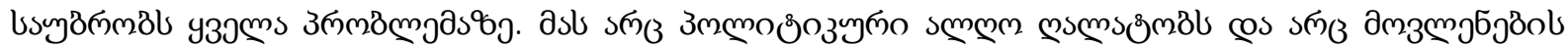

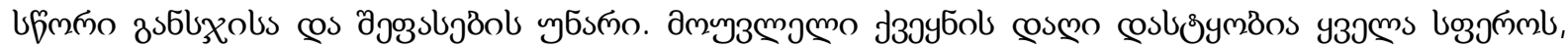

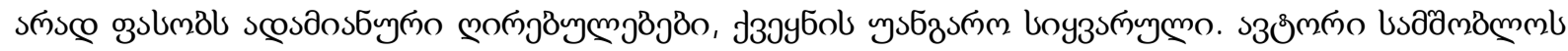

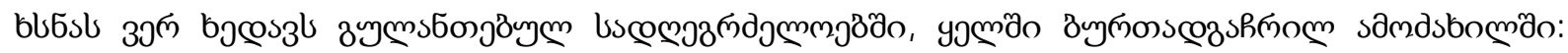

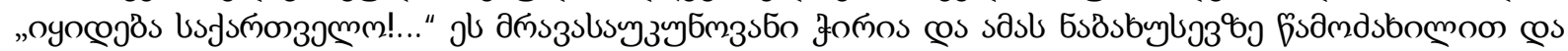

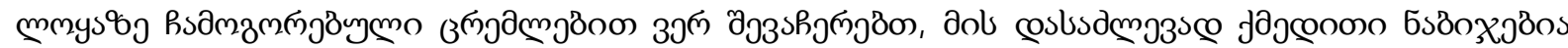
us fonm.

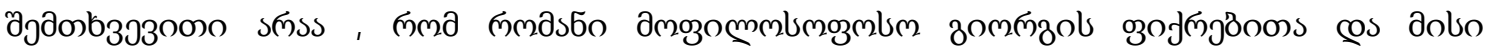

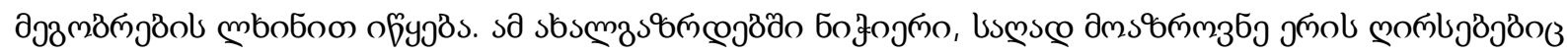

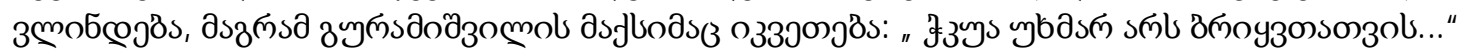

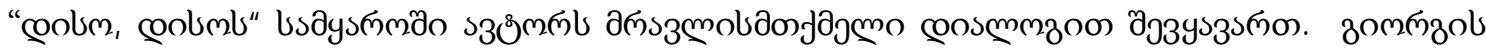

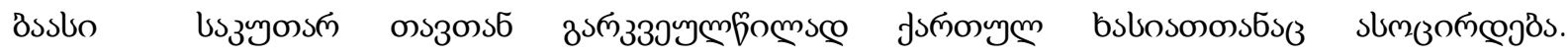

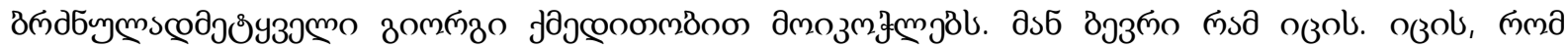

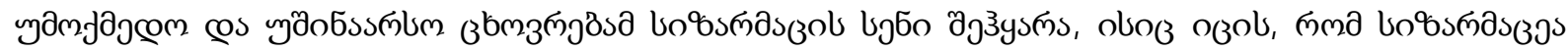

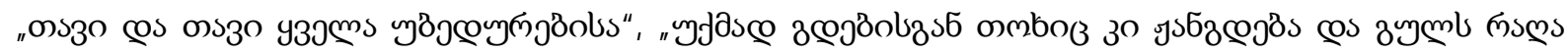

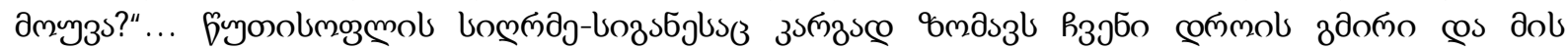

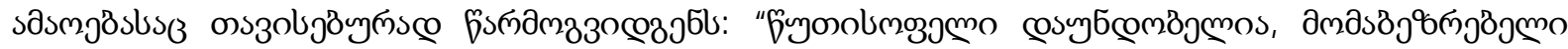

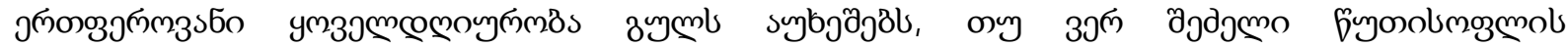

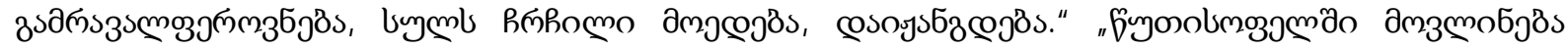

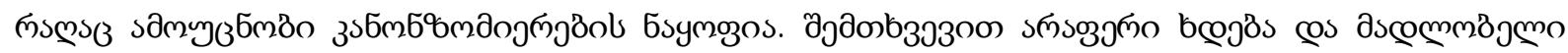

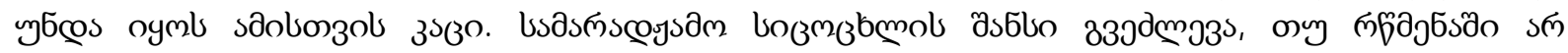

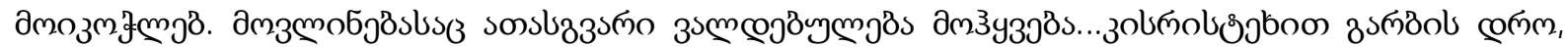

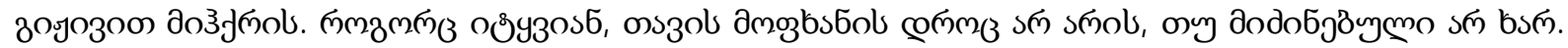

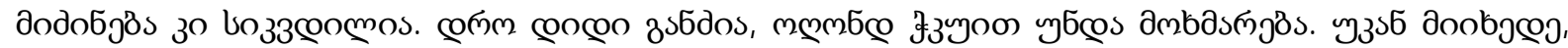

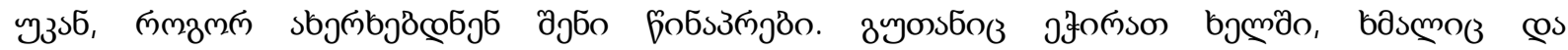

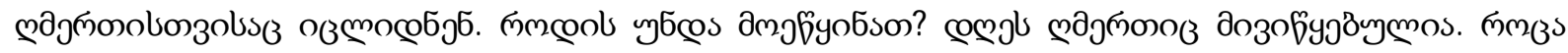

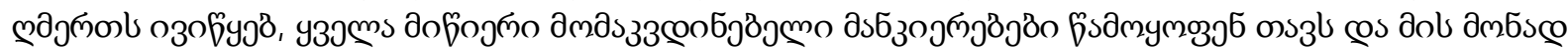

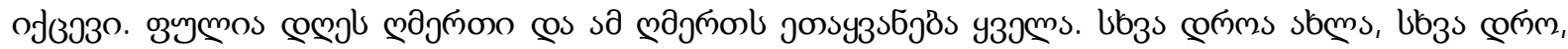

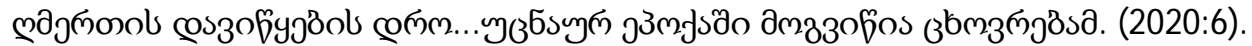

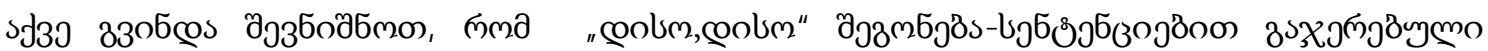

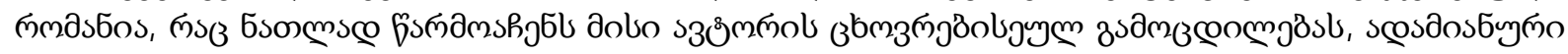

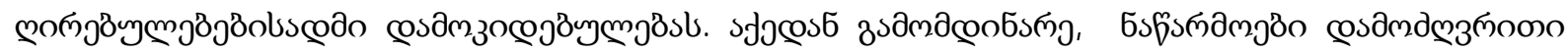

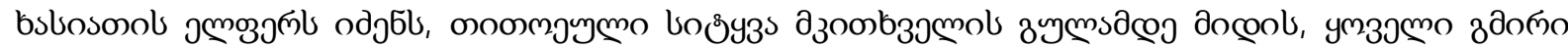

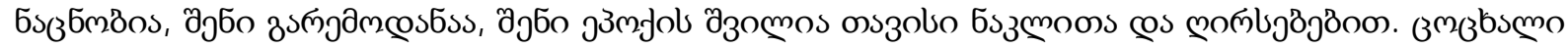

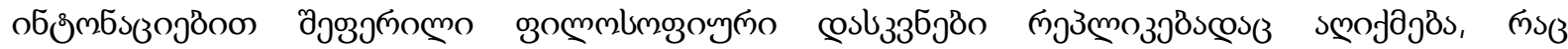

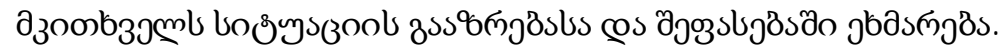

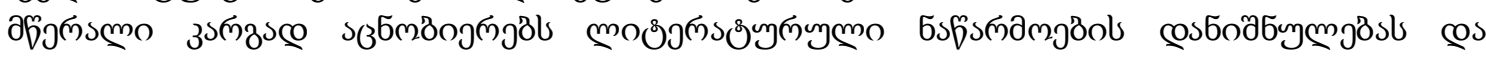

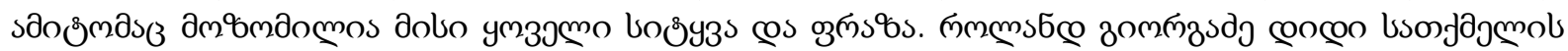




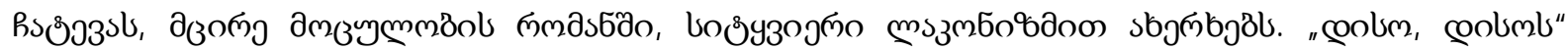

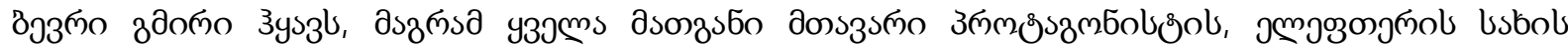

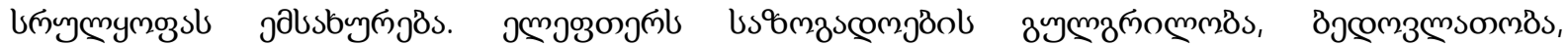

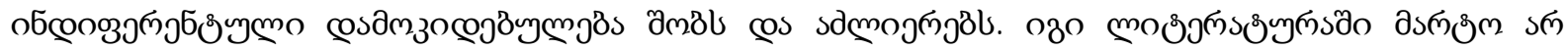

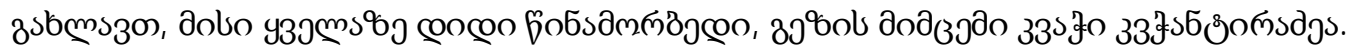

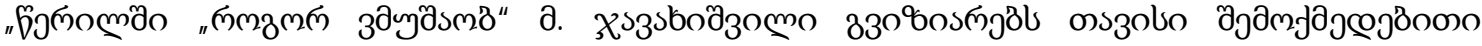

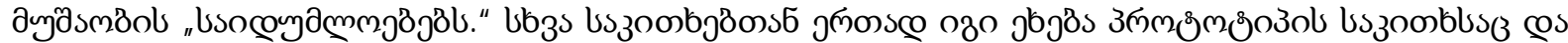

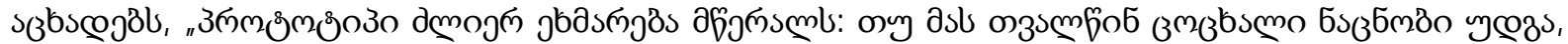

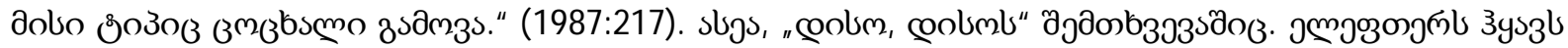

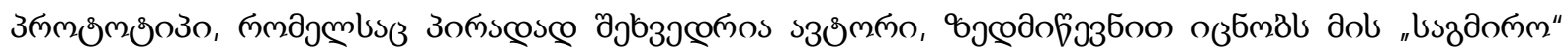

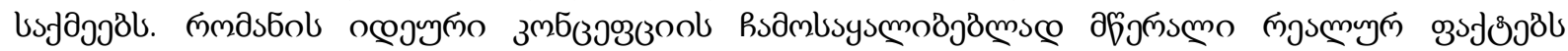

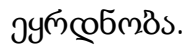

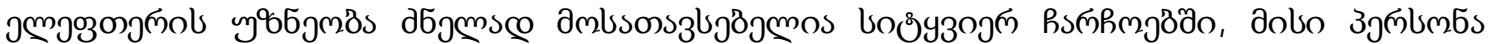

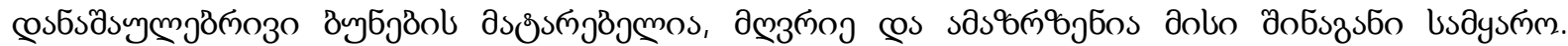

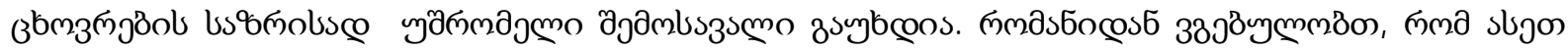

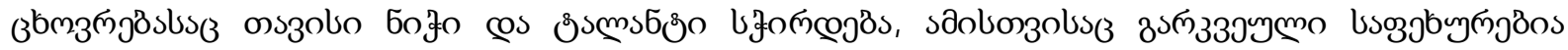

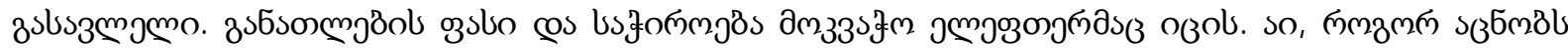

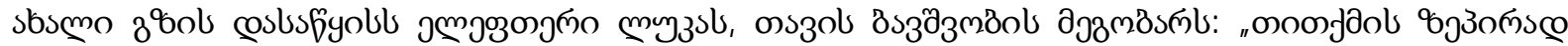

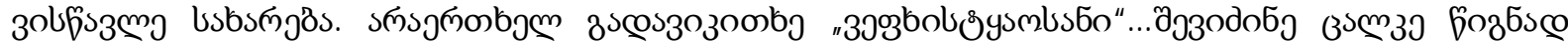

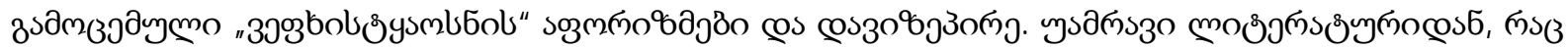

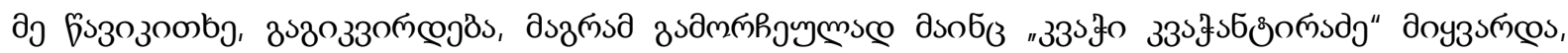

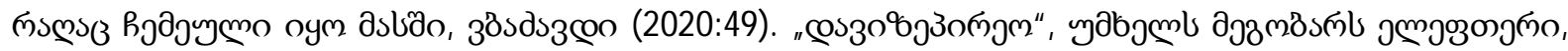

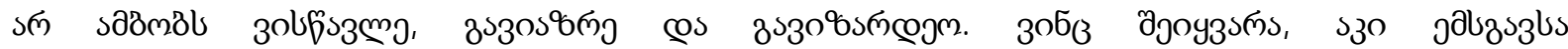

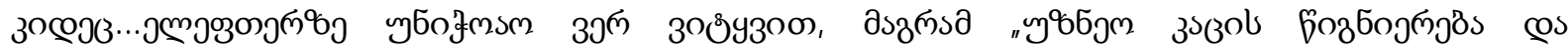

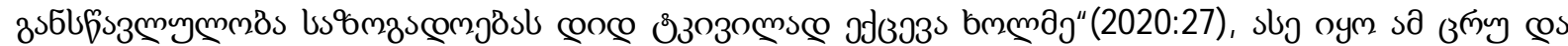

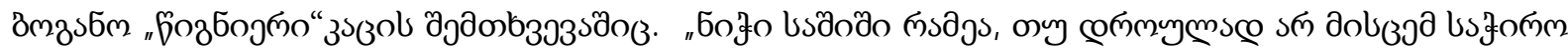

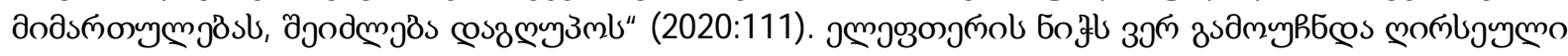

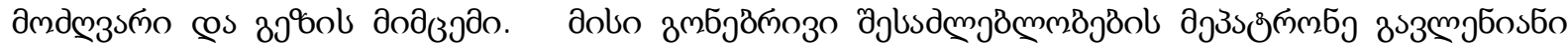

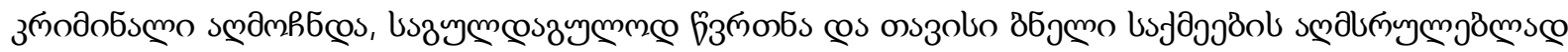
sjoos.

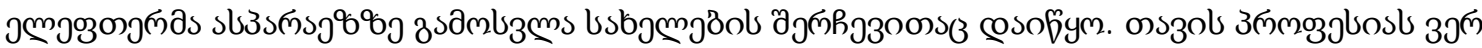

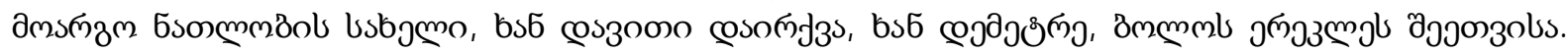

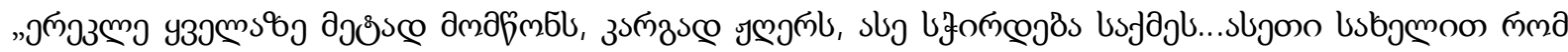

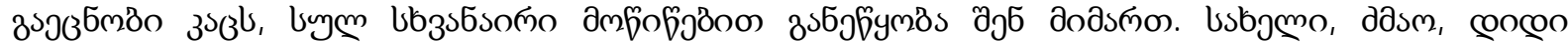

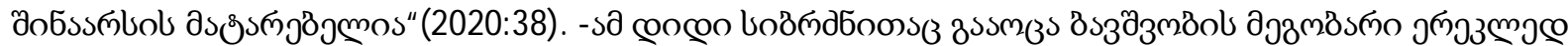

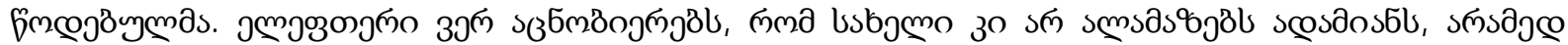

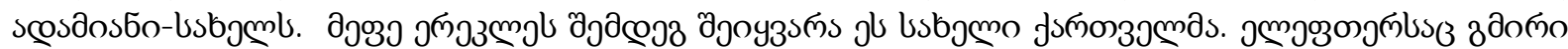

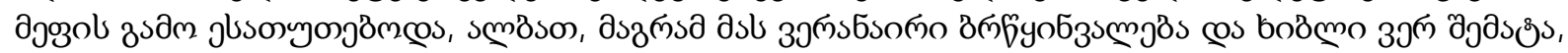

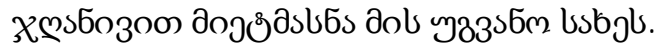

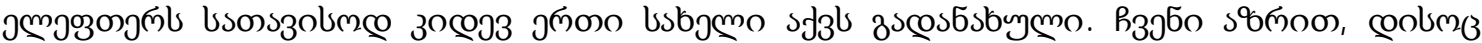

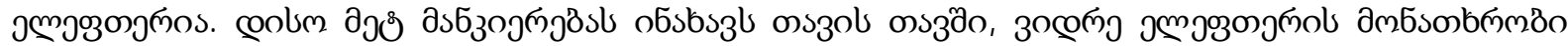

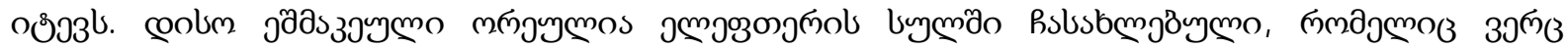

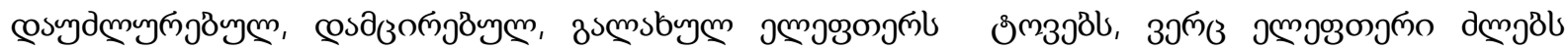

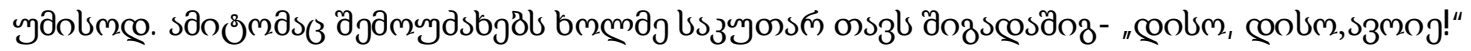

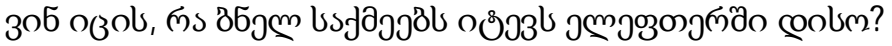

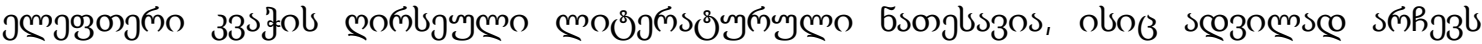

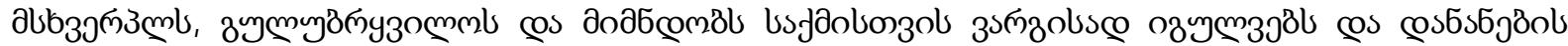

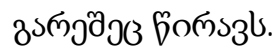

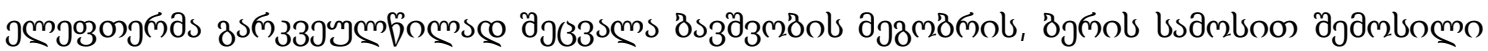

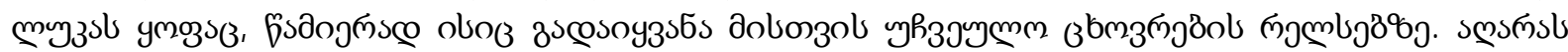




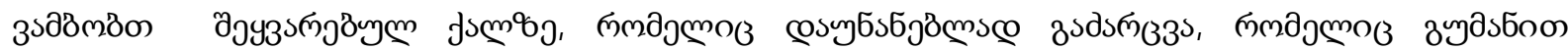

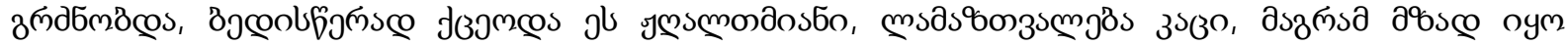

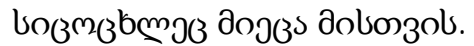

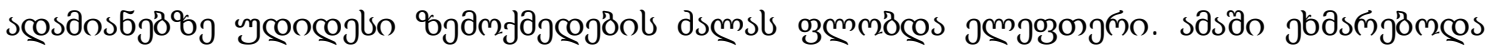

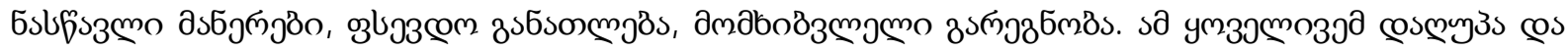

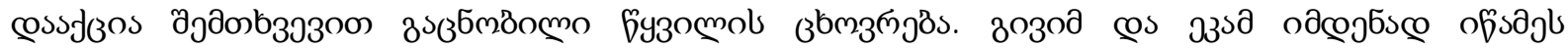

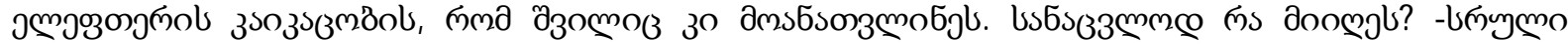

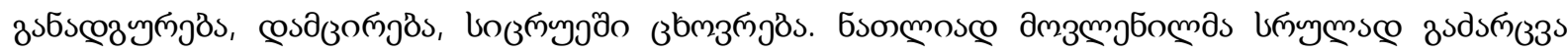

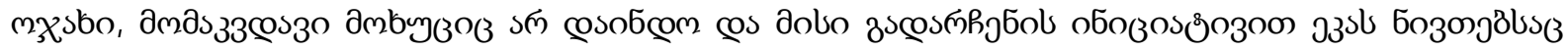

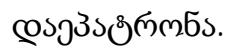

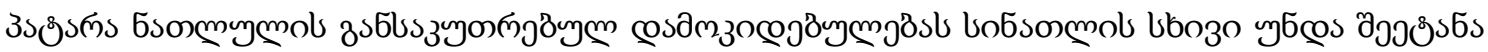

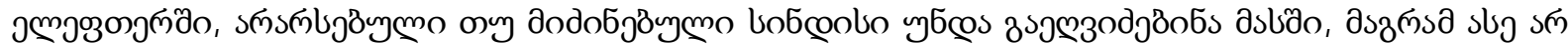

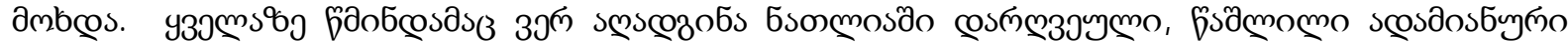

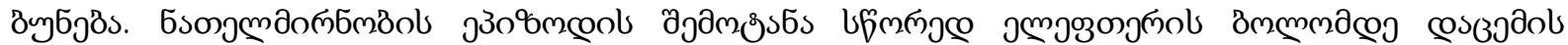

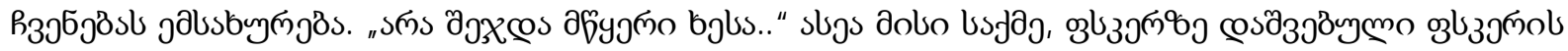

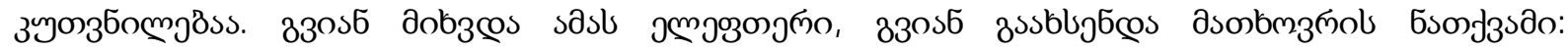

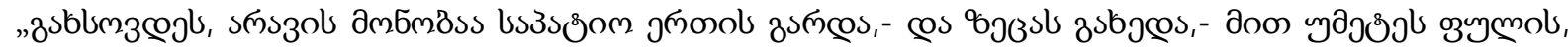

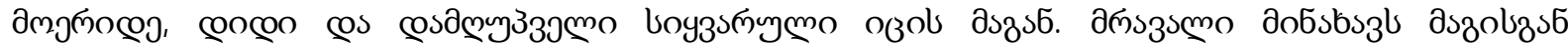

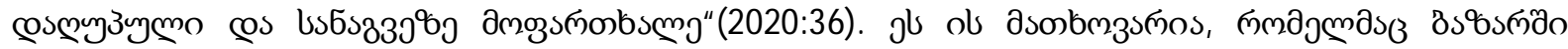

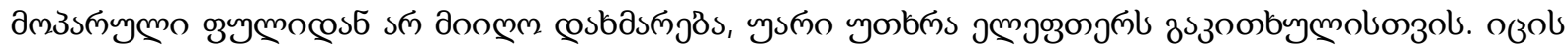

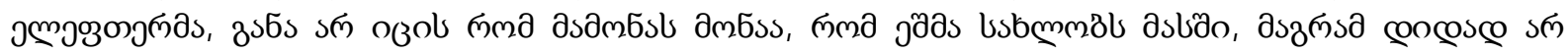

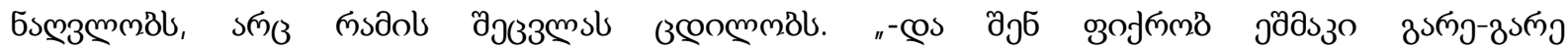

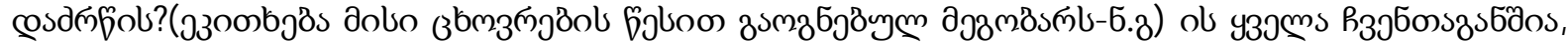

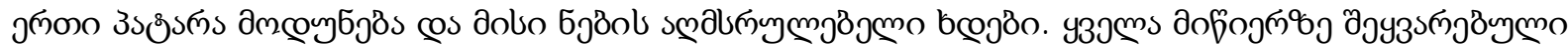

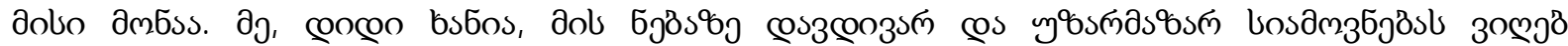

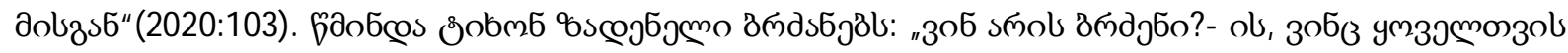

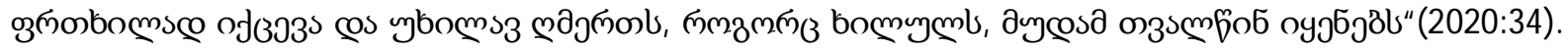

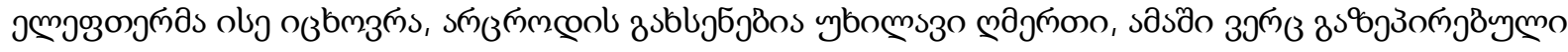
usbsmjass cosjbasms.

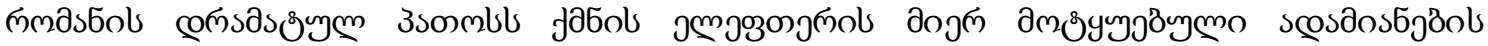

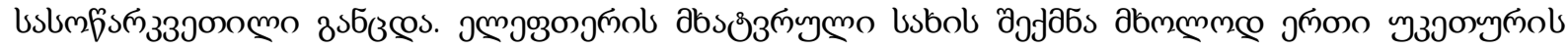

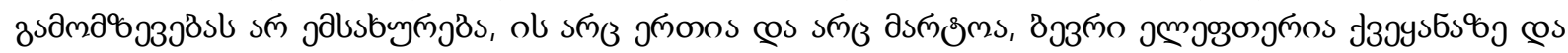

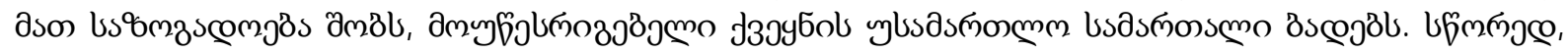

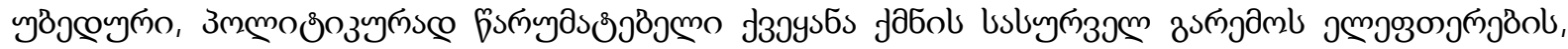

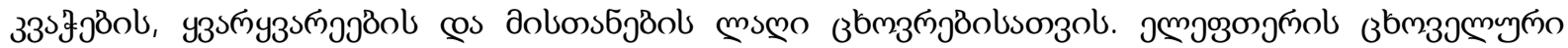

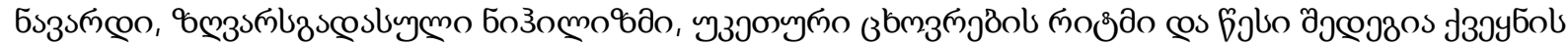

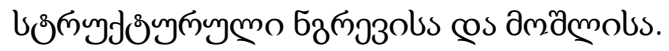

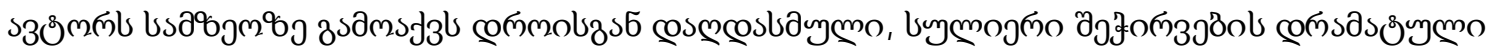

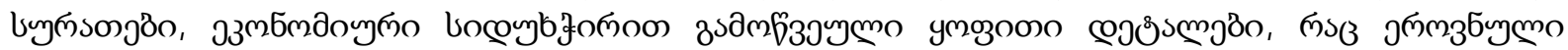

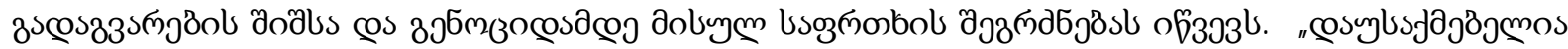

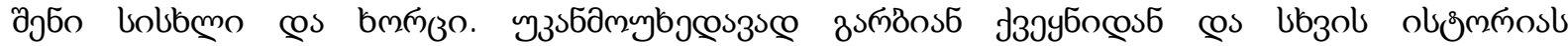

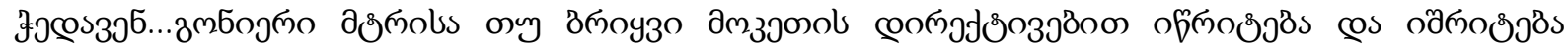

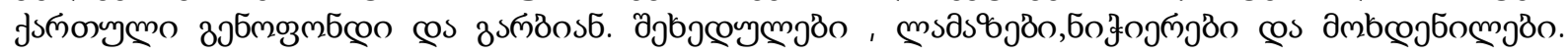

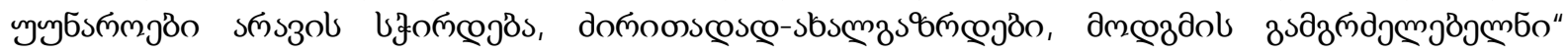

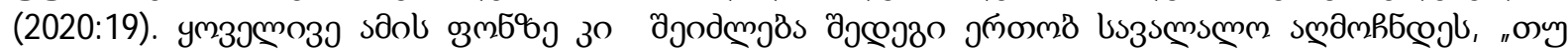

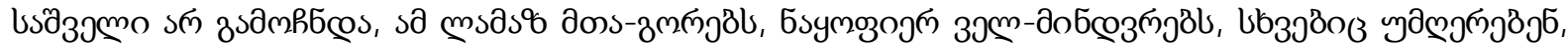

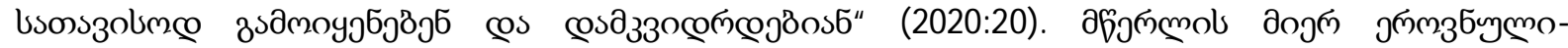

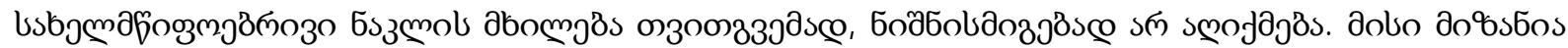

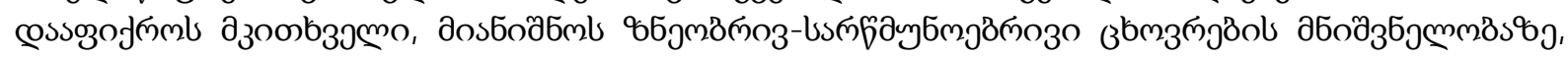

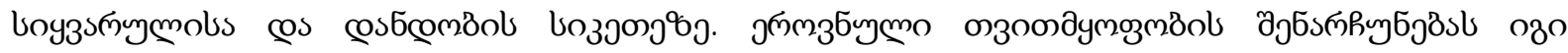




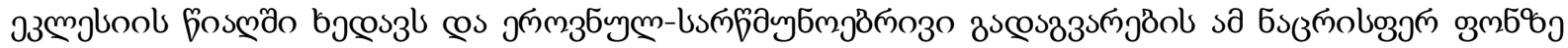

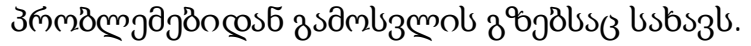

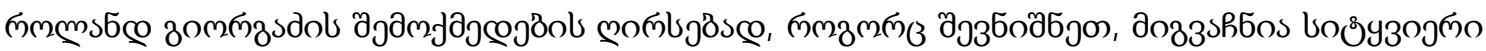

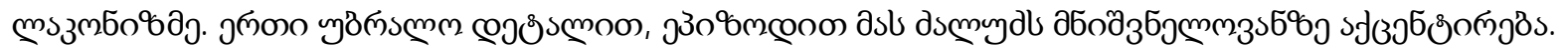

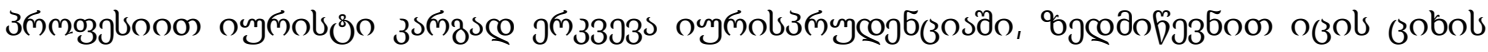

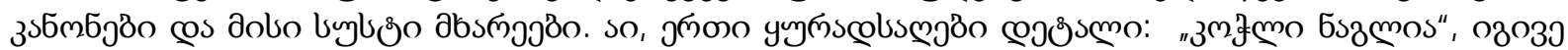

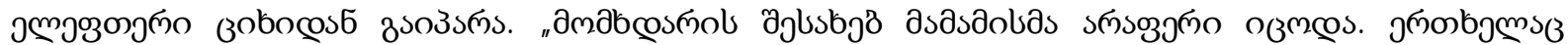

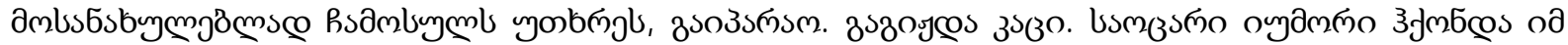

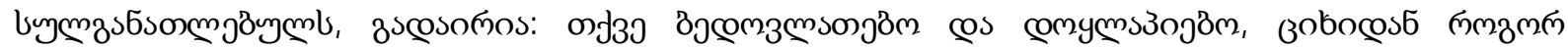

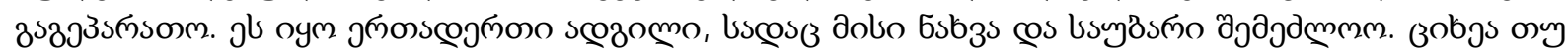

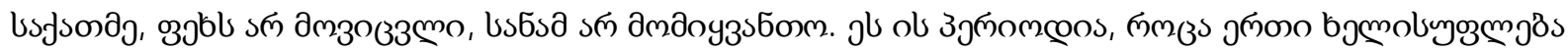

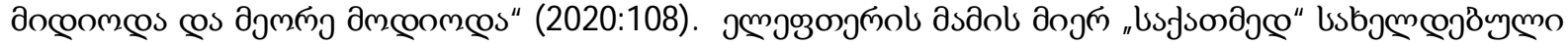

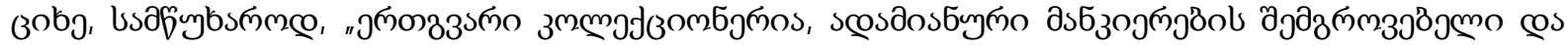

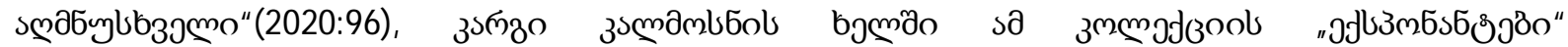

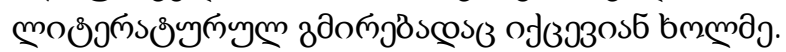

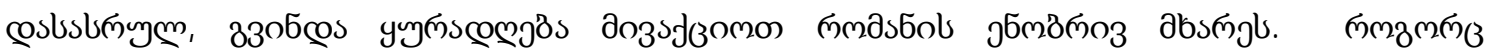

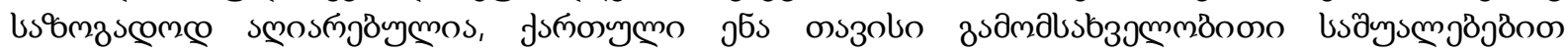

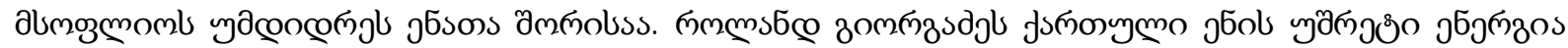

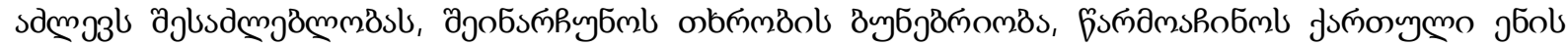

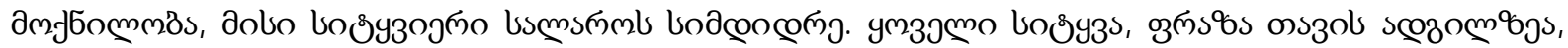

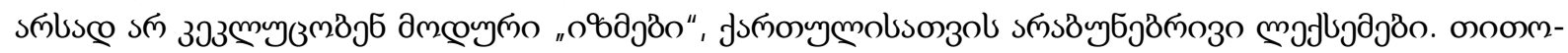

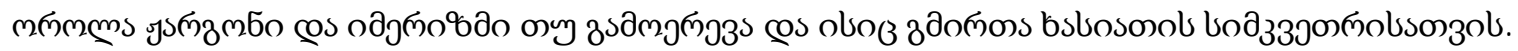

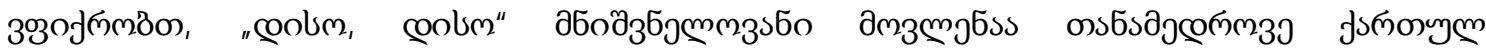

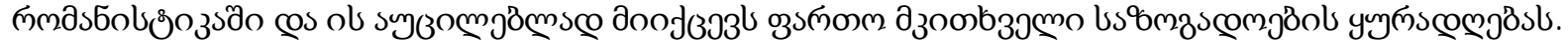

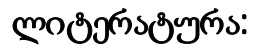

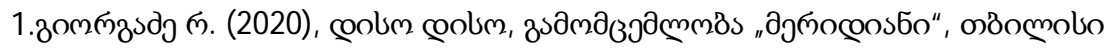

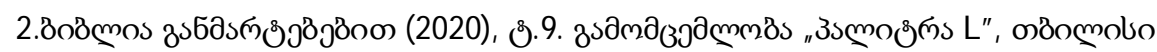

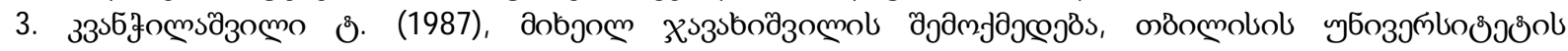

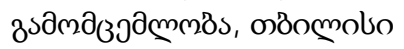

\section{The Old with a Fresh Outlook or the Issue of Protagonists' Identity in Relation to Time}

\author{
Giorgadze Nino \\ Iakob Gogebashvili State University, Telavi
}

\begin{abstract}
Abstract. Roland Giorgadze regularly publishes his poetry and prose in Georgian literary journals and newspapers and thus humbly contributes to the development of Georgian literary processes. The bulk of his work is presented by the following publications: Dream President (2001), The Road (2011), The Cry (2017) and Diso, Diso (2020).

This time we are dealing with the novel Diso, Diso. Here, the writer described life in Georgia in the 90 -ies of the $20^{\text {th }}$ century in gloomy, yet, very realistic colors without any embellishment. Some very accurate stresses are made upon the poor spiritual state of the country and upon the tragic reality it causes. The novel tries to show the deplorable reasons why a human being in this ideal-free society is doomed to become degraded even if they experience a rise (before a fall). The evil has a new beginning and life in every epoch and this new beginning is presented through the character of Elepter in the novel.
\end{abstract}


The novel has a slightly documentary hue. The author openly discusses all the problems. His political flair is infallible and he has a good sense of judging and evaluating current events. All walks of life are branded with the mark of a country not properly attended. Human values and selfless love for one's homeland are no longer priorities.

Diso, Diso is full of didactic maxims that clearly show the life experience of the author, his adherence to lofty values.

Roland Giorgadze understands well the purpose of a literary work and that is why all his words and phrases are loaded with meaning. The author manages to convey a meaningful message is a compressed, verbally laconic way.

The writer uncovers the dramatic scenes of spiritual poverty marked with time, existential details of economical squalor, that cause the fear of national endangerment and the threat of looming genocide. The criticizing and uncovering the national and state faults should not be understood as self-flagellation. It aims at making readers think and also reminding them about the importance of moral life, being close to the creator, about love and the good of forgiving. The author sees the preserving national identity through religious faith. He also sets the ways of coping with these problems in this gloomy scene of national and religious degeneration.

To our judgment, Diso, Diso is an interesting publication on the current literary scene and we hope it will get the attention of both general readers and literary critics.

Key words: Diso, Elepter, didactic maxims, verbal laconism. 